\title{
Using ESG Factors for Equity Valuation
}

By Jeroen Bos, CFA

In the article "Integrating ESG Factors in the Investment Process" (January/February 2014), I discussed the key drivers of the growing importance of integrating environmental, social, and governance (ESG) factors in your investment process. This trend is likely to continue in the coming years because ESG integration in the mainstream investment process provides a clear opportunity to optimize the risk-return characteristics of your portfolio. From a practitioner's perspective, one of the key challenges is how to integrate these factors in a company's valuation, an interesting area that is still in development. Today, investors are still using several different ways of applying ESG factors in company valuations.

Let's begin by focusing on one of the traditional valuation methods, the discounted cash flow (DCF). One of the ways to include ESG is by adjusting the discount rate. Thus, companies that score poorly on ESG metrics will have a higher risk profile on average, and for such companies, one could argue for using a higher discount rate (resulting in a lower valuation) in the DCF. The reverse holds true for companies that score well when it comes to ESG metrics.

Although this method of ESG integration is being used frequently in the sector, it does have two challenges. The first difficulty is the magnitude of adjustment. Should one adjust the discount rate by $25 \mathrm{bps}, 50 \mathrm{bps}$, or maybe even 150 bps? Because research in this field is still very limited, with no clear conclusions yet, the magnitude of adjustment remains an arbitrary decision. The second problem is the risk of double counting. If a company has a higher risk profile as a consequence of relatively poor ESG factors and this risk is already widely known in the market, one could argue that it is already reflected in its discount rate (through a higher company beta, assuming that the capital asset pricing model holds). In such a case, adjusting the discount rate again for ESG factors could be seen as double counting, leading to an unrealistically low fair value for the company. Integrating ESG factors by adjusting the discount rate only works properly if ESG factors are difficult to translate into financials, such as some governance factors, and have not yet been reflected through more or less volatile stock prices.

A better way of integrating ESG factors in the DCF is by adjusting the future cash flows of a company. For example, think about the BP oil spill in the Gulf of Mexico in 2010. Not only did the spill result in fines for the companies involved, but it also led to disruption of production and operations and stricter (hence more expensive) safety measures in the years thereafter. All of these impacts have an effect on future cash flows that can and should be integrated in the DCF. The same could be argued when it comes to the apparel factory collapse in Bangladesh in 2013, which will likely drive costs higher for some of the consumer companies going forward (increasing supply chain costs through better safety standards and potentially higher wages). Another clear example of how ESG factors can drive future cash flows is the potential impact of future water shortages on the operations and profitability of mining companies in certain regions.

The key advantage of this method is that it forces the investor to translate the company's ESG factors into future cash flows and thus to focus on the relevant material issues. That said, it is understandably difficult to estimate cash flow impact on low-probability, high-impact events, such as an oil spill, or to try to assign monetary value to ESG factors for which there is no market, such as governance factors. Still, this way of integration provides a much better starting point for discussion because the assumptions used are clearer than those made in the discount rate adjustment.

In addition to DCF, a widely used and popular way to value companies is multiple analysis. When looking at price/ earnings or price/book multiples, one can integrate ESG factors by adjusting the target multiple. Simply add a premium to the target multiple for companies that do well on ESG and apply a discount to the target multiple for companies that score poorly on this front. These methods have the same issues as the adjustment of the discount rate (arbitrary level of adjustment and risk of double counting). With this way of integration, however, at least an attempt is made to integrate ESG factors so they are not completely absent from the company's valuation and investment conclusion.

Often, trying to translate ESG factors into future cash flows with a high degree of conviction is difficult. For this reason, analysts also need to look at the sensitivities of the ESG factors to the overall valuation and consider different scenarios. These steps will help the investor gain better insights into the materiality and potential impact of ESG factors on the overall valuation. For example, for companies that have a more binary ESG risk, one could look at a "business as usual" scenario and a scenario in which the ESG issues materialize. Both scenarios could be probability weighted to come to a fair value for the company.

The momentum in favor of ESG integration has increased substantially in recent years, a trend that is likely to continue in the years to come. In practice, ESG factors can be integrated in a company's valuation in several different ways, with the translation into future cash flow having the advantage that it provides the most insights and clarity for investors. The discussion on this topic is ongoing, and the field is still evolving. Consensus about the best practice for using ESG factors remains a work in progress.

Jeroen Bos, CFA, is the head of global equity research at ING Investment Management and a member of the board of directors of the CFA Society Netherlands. 